\title{
Dynamique locales de production collective des oranisations de producteurs cotonniers : étude de cas dans le département des Collines au Bénin \\ Local dynamics of collective productionamong cotton producer organizations: A case study in the Collines departmentof Benin
}

\section{Bernard Hounmènou}

Numéro 316, mai 2010

URI : https://id.erudit.org/iderudit/1020896ar

DOI : https://doi.org/10.7202/1020896ar

Aller au sommaire du numéro

Éditeur(s)

Association Recma

ISSN

1626-1682 (imprimé)

2261-2599 (numérique)

Découvrir la revue

Citer cet article

Hounmènou, B. (2010). Dynamique locales de production collective des oranisations de producteurs cotonniers : étude de cas dans le département des Collines au Bénin. Revue internationale de l'économie sociale, (316), 72-84.

https://doi.org/10.7202/1020896ar

\section{Résumé de l'article}

Au Bénin, depuis le début des années 90, la création des groupements villageois (GV) et des unions sous-préfectorales de producteurs (ancienne appellation des unions communales de producteurs, UCP) s'est généralisée notamment autour des activités de production et de commercialisation du coton-graine. Les GV ont pour missions d'assurer l'approvisionnement des membres en intrants, en équipements agricoles et autres facteurs de production, ainsi que l'organisation de la collecte primaire et l'écoulement de la production des membres, et de permettre à ces derniers l'accès au crédit. Mais les GV s'affirment également comme des acteurs déterminants dans la mise en place des biens collectifs au profit de la communauté locale. Les activités du GV mettent en jeu une relation de coopération entre les producteurs cotonniers. La législation béninoise confère à ces groupements ainsi qu'à leurs organisations laîtières un statut d'associations à caractère coopératif. Le dynamisme de ces organisations cotonnières de base a suscité l'organisation d'autres communautés et réseaux de producteurs. 


\section{DYNAMIQUES LOCALES DE PRODUCTION COLLECTIVE DES ORGANISATIONS DE PRODUCTEURS COTONNIERS: ÉTUDE DE CAS DANS LE DÉPARTEMENT DES COLLINES AU BÉNIN}

* Ingénieur agro-économiste, docteur en science économique. Mél. hbenaf@yahoo.fr.

\author{
par Bernard Hounmènou*
}

Au Bénin, depuis le début des années 90, la création des groupements villageois (GV) et des unions sous-préfectorales de producteurs (ancienne appellation des unions communales de producteurs, UCP) s'est généralisée notamment autour des activités de production et de commercialisation du coton-graine. Les GV ont pour missions d'assurer l'approvisionnement des membres en intrants, en équipements agricoles et autres facteurs de production, ainsi que l'organisation de la collecte primaire et l'écoulement de la production des membres, et de permettre à ces derniers l'accès au crédit. Mais les $G V$ s'affirment également comme des acteurs déterminants dans la mise en place des biens collectifs au profit de la communauté locale. Les activités du GV mettent en jeu une relation de coopération entre les producteurs cotonniers. La législation béninoise confère à ces groupements ainsi qu'à leurs organisations faîtières un statut d'associations à caractère coopératif. Le dynamisme de ces organisations cotonnières de base a suscité l'organisation d'autres communautés et réseaux de producteurs.
(1) Ordonnance 59/PR/MDRC du 28 décembre 1966 et son décret d'application $n^{\circ} 516$ 'évolution du mouvement paysan béninois est liée à l'évolution politique du pays. Depuis le début des années 90, la création des groupements villageois $(\mathrm{GV})$ et des unions sous-préfectorales de producteurs (ancienne appellation des unions communales de producteurs, UCP) s'est généralisée notamment autour des activités de production et de commercialisation du coton-graine. Notre hypothèse est que l'essor remarquable de la production cotonnière est intimement lié à l'ancrage territorial de ces organisations et au fait qu'elles mettent en jeu une relation de coopération entre les différents producteurs. La législation béninoise confère en effet à ces groupements ainsi qu'à leurs organisations faîtières un statut d'associations à caractère coopératif ${ }^{(1)}$. Ainsi, au-delà de la poursuite des objectifs de développement agricole, ces organisations collectives se sont investies dans la recherche du bien-être collectif de leurs populations. 
(2) Collecte conduite d'octobre 2002 à mars 2003 et de novembre 2003 à février 2004 dans le cadre d'une préparation de doctorat en sciences économiques sur le thème " Décentralisation, gouvernance participative et dynamiques locales de développement, étude de cas en milieu rural au Bénin " (Hounmènou, 2006).

(3) II s'agit des biens collectifs (équipements, infrastructures ou toute autre réalisation collective bénéficiant à un groupe de personnes). Les biens collectifs sont des biens non exclusifs et non rivaux (Samuelson, 1954).
Après avoir rappelé à grands traits l'histoire contemporaine des groupements villageois (première partie), nous nous attacherons à décrire leurs spécificités. Les GV ont pour missions d'assurer l'approvisionnement des membres en intrants, en équipements agricoles et autres facteurs de production, ainsi que l'organisation de la collecte primaire et l'écoulement de la production des membres, et de permettre à ces derniers l'accès au crédit (deuxième partie). Mais les GV s'affirment également comme des acteurs déterminants dans la mise en place des biens collectifs au profit de la communauté locale (troisième partie). Nous verrons enfin que le dynamisme de ces organisations cotonnières de base a depuis suscité l'organisation d'autres communautés et réseaux de producteurs.

Le présent travail s'appuie sur une collecte de données empiriques auprès des organisations du réseau cotonnier du département des Collines ${ }^{(2)}$. Il s'agit d'enquêtes et d'entretiens réalisés auprès des responsables ou leaders des organisations paysannes et de leurs communautés villageoises. Les enquêtes ont fait usage de questionnaires portant notamment sur des variables liées aux conditions d'identification et de mise en œuvre des initiatives de production collective ${ }^{(3)}$. Les travaux ont plus particulièrement concerné les communes de Glazoué et de Savalou. Dans chacune de ces communes, une dizaine de villages répartis dans cinq arrondissements ont été choisis au hasard pour les enquêtes (deux villages par arrondissement). Dans chacun des villages, l'organisation cotonnière a systématiquement fait l'objet d'investigations. Au total, 38 initiatives des organisations paysannes et 80 des communautés villageoises ont fait l'objet d'enquêtes dans les deux communes. Au-delà des organisations de producteurs villageois enquêtées, les entretiens ont impliqué les unions communales de producteurs de Glazoué, de Savalou et de Dassa-Zoumé (dans les Collines). Ils ont également porté sur l'union départementale des producteurs Zou-Collines et sur la Fédération des unions de producteurs du Bénin.

\section{Emergence des organisations cotonnières}

\section{Organisation coloniale, puis révolutionnaire}

Au Bénin, la filière cotonnière a servi de cadre d'expérimentation à la dynamique organisationnelle du monde paysan. L'émergence des organisations de producteurs remonte à la période coloniale (Berthomé et Pesche, 2003). Au début du $\mathrm{Xx}^{\mathrm{e}}$ siècle, les autorités coloniales ont mis en place des sociétés indigènes de prévoyance qui sont devenues dans les années 50 les sociétés mutuelles de développement rural. Avec l'indépendance en 1960, la coopération agricole devient le modèle de référence. Des textes régissant ces organisations à vocation coopérative sont promulgués en 1961, 1965 et 1966. Dans ce contexte, diverses expériences d'organisations paysannes ont pu se développer dans le pays. En zones cotonnières, ces expériences ont conduit à valider le concept de groupement villageois, 
(4) La nouvelle Constitution béninoise de décembre 1990 reconnaît aux citoyens la liberté d'association, de réunion et de manifestation.

(5) Dans le cadre du programme d'ajustement structurel, le Bénin a été amenéà adopter un programme de restructuration du secteur agricole (PRSA) qui impose le retrait progressif de I'Etat de toutes les activités de production et de commercialisation pour ne se concentrer que sur ses fonctions régaliennes.

(6) En 2003, les travaux ont estimé que le réseau Fupro est composé sur l'ensemble du pays de 6 unions départementales, regroupant elles-mêmes 77 unions communales rassemblant près de 1500 groupements villageois (Berthomé et Pesche, 2003). II existe théoriquement, dans chaque village cotonnier, un groupement villageois qui s'occupe des activités liées à la filière.
(7) Avec la dévaluation du franc de la Communauté financière d'Afrique (FCFA) en 1994, le prix au producteur est passé de 140 FCFA en 1994-1995 à 165, puis à 200 FCFA en 1996-1997. Il a même atteint 225 FCFA en 1998-1999. Depuis lors, il a souvent varié entre 170 et 200 FCFA. Pour la campagne 2009-2010, il a été fixé à 200 FCFA. où des producteurs d'un même village s'associent volontairement pour assumer de multiples fonctions économiques et sociales dans l'intérêt de tous. Ce mouvement, qui a pris forme en zone cotonnière au début des années 70, a été prématurément mis en veilleuse à partir de 1975, lorsque le régime a opté pour "une voie marxiste léniniste ». Durant la période 1975-1990, les groupements villageois ont pu se maintenir tant bien que mal, mais les pouvoirs publics ont promu d'autres formes d'organisation à caractère socialiste, notamment les groupements révolutionnaires à vocation coopérative et les coopératives agricoles expérimentales de type socialiste. Les organisations de ce type étaient fondées sur la lutte des classes, la collectivisation des moyens de production, la modernisation technique... Abandonnées à la fin des années 80 , ces expériences n’ont pas laissé de traces significatives.

\section{Libéralisation économique et structuration de la filière}

Avec le retour du pays à la démocratie à la faveur de la conférence nationale de février $1990^{(4)}$, il est admis que l'Etat ne peut plus être le seul promoteur du développement national. Ce repositionnement a permis en milieu rural la reconnaissance des organisations paysannes comme partenaires du développement. De même, dans le cadre du désengagement de l'Etat des filières de production (et notamment de la filière coton) ${ }^{(5)}$, il a été procédé à une redynamisation des organisations paysannes de base telles que les GV (à partir de 1991), mais aussi à une structuration des producteurs à des niveaux supérieurs, afin d'assurer la coordination des opérations d'approvisionnement et de commercialisation du coton-graine (Oloulotan, 2000). Ce processus, au départ orchestré par l'Etat, s'est autonomisé avec la création des unions départementales des producteurs (UDP) et de la Fédération des unions de producteurs du Bénin (Fupro) en 1994-1995 ${ }^{(6)}$.

Le poids de la filière coton dans l'économie nationale a fait de la Fupro un acteur incontournable. Ceci l'a également obligée à concentrer ses efforts sur la filière coton, dont elle tire l'essentiel de ses ressources. Cette réalité, combinée à de nombreuses difficultés, a conduit à un processus de professionnalisation de l'organisation paysanne dans le secteur cotonnier. Dans la région méridionale du pays qui ne produit pas de coton, les organisations de la Fupro ont une base sociale et des ressources beaucoup plus faibles, ce qui a longtemps constitué un facteur de déséquilibre au sein du réseau. La mise en place du réseau cotonnier est intervenue dans une période de réelle performance du secteur, avec une production qui passe de 20000 tonnes en 1982 à plus de 350000 tonnes à compter de $1996 \mathrm{du}$ fait de la conjonction d'un certain nombre de facteurs tels que l'amélioration du prix au producteur ${ }^{(7)}$, l'augmentation des superficies ensemencées et la hausse des rendements.

\section{L'Association interprofessionnelle du coton}

Dans le processus de libéralisation de la filière cotonnière, la Fupro a conduit des négociations avec d'autres acteurs, en l'occurrence les 
distributeurs d'intrants et les égreneurs. Ces négociations ont abouti à la création de l'Association interprofessionnelle du coton (AIC) en juin 2000. Jusqu'alors, la Société nationale de promotion des produits agricoles (Sonapra) concentrait les opérations de distribution d'intrants et celles d'achat et d'égrenage du coton-graine. Aussi la Centrale de sécurisation de paiement et de recouvrement (CSPR) et la Coopérative d'approvisionnement et de la gestion en intrant agricole ont-elles été mises en place respectivement en 1998 et en 2000 pour mettre fin au monopole de la Sonapra, entreprise publique, sur la gestion de la filière coton. La CSPR assure désormais la gestion des flux physiques et financiers au sein de l'Association interprofessionnelle de coton. La libéralisation de la filière s'est aussi traduite par la mise en place d'une société d'économie mixte pour la gestion de l'outil industriel de la Sonapra (lire l'encadré 1). Au niveau de l'AIC, le manque de respect des règles du jeu par certains acteurs a très tôt entraîné la filière dans une situation caractérisée notamment par une concurrence exacerbée entre égreneurs, la baisse du taux de remboursement du crédit intrant et un surendettement accru des organisations paysannes, avec comme corollaire la perte de confiance entre acteurs de la filière. Ces dysfonctionnements ont eu des répercussions sur la Fupro, avec l'émergence en son sein d'une composante cotonnière.

\section{L'Association nationale des producteurs de coton}

Ces turbulences institutionnelles se sont conjuguées avec la fluctuation des cours mondiaux du coton induite par la surproduction et la subvention des pays producteurs industrialisésà leurs agriculteurs. La Fupro a ainsi dû créer en 2006 une composante cotonnière dénommée Association nationale des producteurs de coton (ANPC). Cette nouvelle structure représente les producteurs de coton du réseau Fupro au sein de l'interprofession (AIC).

\section{Encadré 1 \\ La difficile privatisation de la Sonapra}

Démarré en 1999, le processus de privatisation de la Sonapra a connu de sérieux blocages. Il a fallu attendre juillet 2008 pour qu'il soit réellement relancé et aboutisseà la cession de $33,5 \%$ du capital de la Sonapra à une société privée (Société commune de participation, SCP). Est alors mise en place une société d'économie mixte (laSodeco) dont la structure del'actionnariat comprendl'Etat (33,5\%), laSCP (33,5\%), le publicbéninoisetétranger par lebiais du marché financier régional de l'Union monétaire ouest-africaine (17,5\%), les collectivités locales $(8,5 \%)$, les organisations de producteurs de coton (6\%) et le personnel de la société (1\%). La Sonapra se recentre désormais sur sa mission originelle qu'est la promotion des filières agricoles dans leur ensemble. Une centrale d'achat en intrants agricoles (CAI) est par ailleurs mise en place sous forme de société anonyme, avec participation de l'Etat béninois, des importateurs, des distributeurs d'intrants et de banques. 
Cet ensemble d'éléments a donné lieu dans plusieurs localités du pays à une désaffection de nombre de producteurs. La mise en place de trois familles professionnelles pour prendre en compte les dissidences dans chaque profession a contribué à aplanir les divergences. Ainsi, l'interprofession réunit et coordonne actuellement trois familles professionnelles: le Conseil national des producteurs de coton (CNPC), le Conseil national des égreneurs de coton et le Conseil national des importateurs et distributeurs d'intrants.

Avec plus de $90 \%$ de la production cotonnière du Bénin pour les campagnes 2004-2005 et 2005-2006, l'ANPC s'est taillé une part très confortable dans le Conseil national des producteurs de coton, où ses représentants occupent actuellement tous les postes de président tant sur le plan local (communes et départements) que sur le plan national. En dehors de l'ANPC, les nombreux réseaux de producteurs de coton qui ont vu le jour suite aux dissidences totalisent à peine les $10 \%$ de la production cotonnière pour les deux campagnes de référence.

\section{Dynamique de production des organisations cotonnières}

(8) Cela rejoint les analyses de Soulama (2004) sur les groupements villageois au Burkina Faso.

(9) Lors des entretiens, les responsables paysans ont à titre indicatif affirmé dans les Collines qu'il pourrait varier de 70 à 450 .

(10) Aux niveaux départemental et national, cette coordination relevait des responsabilités respectives des unions départementales des producteurs et de la Fédération des unions des producteurs du Bénin. Avec les récentes réformes de libéralisation de la filière, cette coordination est assurée au niveau national par I'ANPC (pour le compte de la Fupro), au sein du CNPC regroupant plusieurs réseaux de producteurs.
Les GV ont d'abord et exclusivement émergé dans les localités cotonnières. La dynamique de production collective des groupements villageois y revêt une double dimension. La première est liée à la fourniture de services de distribution d'intrants agricoles et de commercialisation du coton-graine aux producteurs cotonniers. La deuxième dimension est relative à la participation du GV à la production de biens collectifs destinés à l'usage de toute la communauté villageoise ${ }^{(8)}$.

\section{Production et commercialisation}

Dès le départ, le groupement villageois est considéré comme une organisation à l'intérieur de laquelle les paysans peuvent assurer eux-mêmes la gestion des services liés à leur production. L'effectif des membres du GV varie suivant la taille du village ${ }^{(9)}$. A l'intérieur des GV, les paysans du village organisent collectivement la distribution des intrants après réception des commandes. Lorganisation de la commercialisation primaire du coton-graine constitue une importante activité à laquelle les groupements villageois se sont habitués après leur création. En effet, à travers les « marchés autogérés ", les producteurs du village réalisent collectivement la pesée et le gardiennage du coton-graine avant son acheminement vers les usines d'égrenage. Les groupements villageois, à travers leurs secrétaires, tiennent des documents de suivi des achats et des évacuations de cotongraine. Ces données leur permettent de procéder au paiement des producteurs après prélèvement des crédits intrants.

La coordination et le suivi de ces opérations à l'échelle communale sont assurés par l'union communale des producteurs (UCP) de coton ${ }^{(10)}$. Outre la coordination des marchés autogérés, cette union fournit aux 
(11) Plusieurs règlements intérieurs de GV indiquent qu'en dehors des droits d'adhésion, des parts sociales libérées, des cotisations spéciales des membres, des frais de prestation de services, de dif férentes réserves constituées... les ressources de I'organisation consistent éventuellement en des prélèvements opérés sur les ristournes et les plus-values des membres. groupements villageois d'importants services tels que le cautionnement des crédits de campagne (lire l'encadré 2) et la coordination des opérations de distribution d'intrants agricoles.

\section{Des ristournes pour financer des investissements collectifs}

Les organisations cotonnières tirent d'importantes ressources des marchés autogérés. Le suivi de la pesée du coton sur ces marchés par les représentants des GV et de l'union communale permet d'évaluer et de répartir les écarts de poids constatés entre le village et l'usine. Les écarts positifs de poids donnent lieu en fin de campagne de commercialisation à des ristournes versées aux producteurs villageois, au prorata de leurs ventes respectives. Dans plusieurs villages, ces ristournes sont en partie ou entièrement orientées par le GV vers des investissements collectifs. Ce principe de ristourne n'est pas une obligation statutaire des GV. Il fait plutôt partie des règles associées à l'organisation de la commercialisation du coton-graine au niveau national. La décision de les orienter en partie dans les investissements collectifs peut toutefois être prise de façon souveraine par les membres d'un GV, en fonction des règles de fonctionnement propres à l'organisation ${ }^{(11)}$.

Les services collectifs fournis par les GV et les organisations faittières lors des opérations de distribution d'intrants et de commercialisation de coton-graine leur donnent droit à des frais de prestation qui leur sont payés en fin de campagne (voir les estimations de ressources individuelles des producteurs et de ressources collectives du GV et de l'UCP présentées en annexe). Les frais de prestation des GV sont aussi orientés vers des investissements collectifs des villageois.

Les ressources collectives générées par les activités cotonnières permettent aux GV de financer des équipements à l'usage de leurs membres. Ces

\section{Encadré 2}

\section{Les groupes de caution solidaire}

Le principe de caution solidaire au sein des GV a récemment été révisé du fait des difficultés récurrentes de remboursement de crédit et des déconvenues souvent subies par les bons payeurs. Actuellement, le GV ne cautionne plus individuellement les producteurs. Il le fait à travers des groupes de caution solidaire (GCS). Ce sont des groupes restreints de producteurs (quatre à sept membres d'un même GV) désireux d'intensifier leur production et qui s'engagent à se porter mutuellement caution afin d'assurer le remboursement. Le président du groupe recense les besoins des membres en qui il a confiance. Ces besoins sont portés au niveau du conseil d'administration du GV, qui les étudie avant de les transmettre à l'UCP. C'est sur la base de ces données que l'UCP lance sa commande d'intrants. A l'approvisionnement des intrants, l'UCP répartit les quantités de chaque GV, qui à son tour les redistribue aux GCS. Chaque GCS à son niveau sert ses membres. 
(12) Quelques GV (une minorité) s'équipent de bascule. Dans leur majorité, ils procèdent à la location de cet équipement pour des pesées sur les marchés auto-gérés de coton-graine. équipements sont constitués dans une grande mesure de magasins de stockage d'intrants ${ }^{(12)}$. Tout comme les services de distribution d'intrants et de commercialisation de coton-graine, il s'agit de biens destinés à la consommation exclusive des producteurs membres de GV. Certaines unions communales réalisent aussi grâce aux ressources cotonnières des actions de production de biens collectifs au profit des producteurs de leur territoire. Ces actions concernent surtout la construction de sièges (bureaux et salles de réunion) et l'acquisition de matériels roulants (véhicules légers, camions).

La mise en place des organisations du réseau des groupements villageois, dans le cadre du transfert par les institutions étatiques de certaines compétences en matière de production et de gestion de services agricoles, met en relief un mécanisme de décentralisation sectorielle, en particulier dans les localités cotonnières. Ce mécanisme a favorisé l'émergence d'un cadre de proximité (système associatif) dans la fourniture de biens collectifs en milieu rural. Le fonctionnement du système donne lieu au niveau des villages et des communes à une double dynamique collective de production. La première permet une fourniture exclusive de services aux membres du réseau. La deuxième consiste en la production de biens à l'usage des populations des territoires en cause. En créant chez les populations des localités concernées une habitude de participation aux initiatives de production de biens collectifs, la dynamique cotonnière favorise l'apprentissage de l'action collective.

\section{Un acteur déterminant dans la mise en place des biens collectifs au profit de la communauté locale}

L'étude de la dynamique de production collective villageoise met en relief un rôle déterminant des organisations cotonnières tant au niveau du financement que de la prise de décisions relatives à cette production.

\section{Les GV, pivot de l'investissement}

L'étude du mécanisme de financement de la production de biens collectifs dans les villages cotonniers révèle que la participation locale à cette production est favorisée par le dynamisme des GV. Les subventions des groupements villageois constituent dans ces localités la forme de participation financière locale la plus fréquente. Cette participation intervient respectivement dans 43 et $38 \%$ des initiatives mises en œuvre par les communautés villageoises des communes de Savalou et de Glazoué (Hounmènou, 2006).

Les prestations des GV sur la distribution des intrants et la commercialisation du coton-graine leur permettent en effet de mobiliser d'importantes ressources financières, qu'ils orientent en partie dans le financement des initiatives de leur communauté villageoise. Par ailleurs, les recettes et les ristournes cotonnières constituent dans ces villages 
les principales sources à travers lesquelles les contributions des ménages (cotisations) sont mobilisées. La collecte de ces contributions dans les villages cotonniers est favorisée par des paiements groupés des recettes cotonnières aux paysans, par le biais de leur GV. A l'occasion de ces paiements, le GV prélève lesdites cotisations sur les avoirs de chaque producteur cotonnier (paiement à la source). Ce rôle déterminant des GV dans le financement de la production collective villageoise fait d'eux des acteurs décisifs de cette production. Non seulement ces organisations sont à l'origine d'une bonne partie des initiatives de leur localité, mais l'avis favorable de leurs membres reste déterminant pour la conduite d'initiatives émanant d'autres types d'acteurs du territoire villageois. Cela fait des GV des interlocuteurs crédibles de nombreux partenaires dans le processus de choix et de mise en œuvre d'initiatives de développement dans les localités cotonnières.

\section{Les réalisations communautaires des GV}

Les ressources collectives des GV leur permettent d'intervenir dans le financement des initiatives de production collective de leur population. Les actions à travers lesquelles les GV participent à cette production sont en particulier celles relatives à la mise en place des infrastructures sociocommunautaires. Dans les communes de Glazoué et de Savalou, ces actions ont concerné la construction d'infrastructures scolaires et sanitaires, l'aménagement d'un marché et de pistes de desserte rurale, la mise en place d'équipements de fourniture d'eau potable, l'installation de caisses d'épargne-crédit, etc. (voir le tableau 1 , en page suivante).

Au-delà des biens produits au sein des GV pour la consommation exclusive des membres, ces organisations s'impliquent dans des initiatives qui profitent aux populations de leur milieu. Dans nombre de localités, elles arrivent à cofinancer des réalisations initiées par les populations avec l'appui d'autres partenaires (pouvoirs publics, ONG, bailleurs...) dans le domaine de la production de biens collectifs. Cette situation a suscité un intérêt relativement important des partenaires, notamment les bailleurs de fonds cherchant des cofinancements.

Tout comme les groupements villageois, les unions communales de producteurs arrivent à participer dans nombre de localités cotonnières

(13) A Glazoué par exemple, I'union communale des producteurs avait participé en 1997 au financement de la mise en place d'une radio communautaire, Dassa-Glazoué, devant desservir les populations de son territoire. au financement de la production collective des populations de leur territoire ${ }^{(13)}$. Les actions à travers lesquelles les unions communales participent à ce type de production concernent souvent la mise en place d'équipements à caractère communal ou intervillageois. Les réalisations collectives des GV et des unions de producteurs interviennent souvent à l'issue d'un processus décisionnel collectif. L'implication de ces organisations dans le développement local les amène à mobiliser des ressources bénévoles (participation des membres), des ressources marchandes (revenus d'activité) et parfois des ressources non marchandes (subventions éventuelles des partenaires). 
Tableau 1

Interventions des GV dans la mise en œuvre des initiatives locales de production de biens collectifs

\begin{tabular}{|c|c|c|c|c|c|}
\hline Commune & Arrondissement & $\begin{array}{l}\text { Localisation } \\
\text { du/des GV }\end{array}$ & Actions concernées & Appui du GV & Année \\
\hline \multirow{3}{*}{ Savalou } & \multirow[b]{2}{*}{ Lahotan } & \multirow[b]{2}{*}{$\begin{array}{l}\text { Tous } \\
\text { les villages }\end{array}$} & $\begin{array}{l}\text { Installation de réseau } \\
\text { d'adduction d'eau }\end{array}$ & $\begin{array}{l}\text { Cofinancement } \\
(400000 \text { FCFA })\end{array}$ & 1998 \\
\hline & & & \begin{tabular}{|l|} 
Aménagement \\
de la voie Koutago- \\
Lahotan
\end{tabular} & $\begin{array}{l}\text { Aide } \\
\text { à la mobilisation } \\
\text { du cofinancement }\end{array}$ & 1999 \\
\hline & Ouèssè & Tchogodo & $\begin{array}{l}\text { Construction } \\
\text { du local d'une caisse } \\
\text { d'épargne-crédit }\end{array}$ & $\begin{array}{l}\text { Cofinancement } \\
\text { (300 000 FCFA) }\end{array}$ & 2001 \\
\hline \multirow{4}{*}{ Glazoué } & \multirow[t]{2}{*}{ Ouèdèmè } & Kpota & \begin{tabular}{|l|} 
Installation \\
d'une caisse rurale \\
d'épargne et de prêt \\
(CREP) \\
\end{tabular} & $\begin{array}{l}\text { Cofinancement } \\
\text { (100 000 FCFA) }\end{array}$ & 1996 \\
\hline & & Yagbo & $\begin{array}{l}\text { Aménagement de } \\
\text { hangars au marché }\end{array}$ & $\begin{array}{l}\text { Cofinancement } \\
(120 \text { 000 FCFA) }\end{array}$ & 1998 \\
\hline & Gomé & Gomé & $\begin{array}{l}\text { Réhabilitation } \\
\text { des bureaux } \\
\text { de l'arrondissement }\end{array}$ & Cofinancement & 2002 \\
\hline & Magoumi & Magoumi & $\begin{array}{l}\text { Construction de } \\
\text { module de classe } \\
\text { pour le collège de } \\
\text { l'arrondissement }\end{array}$ & Cofinancement & 2004 \\
\hline
\end{tabular}

Source: Hounmènou, 2006.

\section{Organisation et leadership des groupements villageois}

Au sein des groupements villageois et des unions communales de producteurs, la coordination des activités est assurée par un conseil d’administration constitué de dirigeants élus par les membres en assemblée générale. Au niveau villageois, il s'agit des producteurs cotonniers ayant adhéré à l'organisation. Au niveau communal, l'assemblée regroupe les délégués des GV issus de leur conseil d'administration. Les textes fondamentaux des GV et ceux des UCP prévoient que les décisions en matière de conduite d'action collective soient initiées par les membres du conseil d'administration et soumises à la validation de l'assemblée. Ces décisions doivent être prises à la majorité démocratique suivant le principe "Une personne, une voix ». Les propositions d'initiatives sont 
(14) Les leaders désignent ici les personnes démocratiquement désignées par les adhérents des GV pour coordonner leurs activités. II s'agit en parti-culier des présidents, assistés des autres membres de l'organe de coordination.

(15) Toutefois, au-delà des leaders, les initiatives collectives des GV proviennent dans une moindre mesure d'autres membres $(23$ et $25 \%$ des cas étudiés respectivement à Savalou et Glazoué), notamment lors des diagnostics de développement des organisations. d'abord étudiées à l'intérieur du conseil. En cas de décision favorable, les actions à mener sont planifiées et inscrites au programme d'activité de l'organisation, mais ce n'est qu'après la décision favorable de l'assemblée générale qu'elles sont réalisées. Si au niveau de l'union communale des producteurs le mode de décision le plus courant est le vote, la recherche du consensus apparaît privilégiée dans un premier temps au niveau du groupement villageois. Ceci est particulièrement valable pour des décisions relatives aux initiatives des groupements en faveur de l'amélioration du bien-être collectif de l'ensemble de la communauté.

Sur les territoires cotonniers de Glazoué et de Savalou, les initiatives collectives des communautés émergent des groupements villageois respectivement dans au moins 15 et $22 \%$ des cas. Dans le choix des initiatives collectives au sein de ces organisations, leurs leaders (ou responsables) jouent un rôle important ${ }^{(14)}$. En effet, 56 \% des initiatives mises en œuvre par les organisations cotonnières étudiées proviennent de ces leaders. En tant que porte-parole des organisations, ils constituent de réelles forces de proposition en matière d'initiatives collectives de production ${ }^{(15)}$. Toutefois, les initiatives mises en œuvre par les GV sont révélées essentiellement lors des concertations réunissant leurs membres $(82 \%$ des cas étudiés). Dans la procédure de décision, les choix collectifs interviennent essentiellement à travers le consensus (65\% des cas) ou par unanimité des membres (35\% des cas).

\section{Conclusion}

Du fait d'une structuration et d'un important développement de la production cotonnière au début des années 90 , les organisations villageoises mises en place pour la gestion de la filière ont fait l'objet d'un réel dynamisme dans la production de bien-être collectif dans leur milieu. Sur la période étudiée, elles ont donné lieu à une double fonction de production. La première permet la fourniture de services aux membres des organisations du réseau. La deuxième donne lieu à une production de biens collectifs à l'usage de la communauté locale. A travers cette deuxième dynamique, les organisations cotonnières ont joué un rôle éminent non seulement dans le financement de leur localité, mais également dans les prises de décisions relatives à cette production. Cet élément constitue un avantage spécifique des localités cotonnières et positionne aussi les GV comme des partenaires importants des communes actuelles dans la dynamique de développement local.

Cette propriété des GV peut être valorisée dans le cadre des responsabilités des communes béninoises actuelles. Les GV pourraient en effet servir de relais aux communes pour certaines initiatives en faveur des villages, notamment celles relatives à la promotion de l'agriculture locale. Il serait donc opportun d'étudier dans quelle mesure les communes peuvent s'articuler avec les GV, par exemple dans le cadre d'une maîtrise d'ouvrage 
déléguée, en vue d'appuyer des initiatives en faveur du secteur conformément aux orientations actuelles de la stratégie nationale du conseil agricole au Bénin. Les exigences d'une responsabilisation des communes dans l'actuel contexte de décentralisation cumulées avec leur défaillance en compétences techniques dans le secteur agricole rendent nécessaire cette délégation de fonction pour laquelle elles sont appelées à rester maîtres d'ouvrage (Hounmènou, 2008).

\section{Bibliographie}

Berthomé J., Pesche D., 2003, Analyse et capitalisation des démarches d'appui aux organisations de producteurs $(O P)$ de la coopération française (MAE et AFD), Bénin, ministère des Affaires étrangères, DGCID/DCT-EPS, $39 \mathrm{p}$. Hounmènou B., 2008, Décentralisation et partenariat public-privé: une contribution à l'amélioration de la gouvernance du secteur agricole au Bénin, communication au IV $\mathrm{V}^{\mathrm{e}}$ colloque international du réseau Syal (Système agroalimentaire localisé), "Alimentation, agriculture familiale et territoire ", Mar del Plata (Argentine), 27-30 octobre, $22 \mathrm{p}$.

Hounmènou B., 2006, "Décentralisation, gouvernance participative et dynamiques locales de développement, étude de cas en milieu rural au Bénin ", thèse de doctorat en sciences économiques, université de Versailles Saint-Quentin-en-Yvelines, 379 p.

Hounmènou B., 2004, Dynamique des organisations paysannes et développement local au
Bénin, communication au congrès international "Agro-industrie rurale et territoire " (Arte), Toluca, Etat de Mexico, Mexique, $1^{\text {er-}-4}$ décembre, $21 \mathrm{p}$.

Inter-Réseaux, 2008, L'Association interprofessionnelle du coton au Bénin, septembre, $13 \mathrm{p}$.

Oloulotan S., 2000, "Les OP des zones cotonnières du Bénin ", Grain de sel (InterRéseaux), nº 18, juillet 2001, p. 16-17.

Samuelson P. A., 1954, "The pure theory of public expenditure ", The Review of economics and statistic, 36, p. 387-389.

SoulamaS., 2004, « Le groupement villageois au Burkina Faso: une organisation d'économie sociale? » in Charlier S., Nyssens M., Peemans J.-P. et Del Castillo I. Y. (dir.), Une solidarité en acte: gouvernance locale, économie sociale, pratiques populaires face à la globalisation, Presses universitaires de Louvain, Belgique, p. 148-173. 
Annexe

Indications relatives aux revenus cotonniers:

le cas du département des Collines

\begin{abstract}
Revenu individuel
Le producteur cotonnier moyen réalise dans les Collines une superficie de 3 hectares de coton par an. Sur la base d'un rendement moyen de 1,5 tonne de coton-graine à l'hectare et d'un prix bord champ de 190000 francs de la Communauté financière d'Afrique (FCFA) la tonne, la marge du producteur est présentée dans le tableau A.
\end{abstract}

\title{
Tableau A
}

Estimation de la marge individuelle du producteur cotonnier dans les Collines

\begin{tabular}{|l|l|r|r|}
\cline { 3 - 4 } \multicolumn{2}{c|}{} & $\begin{array}{r}\text { Estimation } \\
\text { à l'hectare }\end{array}$ & $\begin{array}{r}\text { Estimation pour } \\
\text { le producteur moyen }\end{array}$ \\
\hline \multirow{4}{*}{ Production } & Quantité (tonnes) & 1,5 & 4,5 \\
\cline { 2 - 4 } & Valeur monétaire (FCFA) & $\mathbf{2 8 5 0 0 0}$ & $\mathbf{8 5 5 0 0 0}$ \\
\hline \multirow{4}{*}{$\begin{array}{l}\text { Coût de production } \\
\text { (FCFA) }\end{array}$} & Intrant engrais & 40000 & 120000 \\
\cline { 2 - 4 } & Intrant insecticide & 49200 & 147600 \\
\cline { 2 - 4 } & Main-d'ouvre & $\mathbf{1 5 0 0 0 0}$ & $\mathbf{4 5 0 0 0 0}$ \\
\cline { 2 - 4 } & Coût total & 194920 & 584760 \\
\hline Marge brute (FCFA) & & 90080 & 270240 \\
\hline
\end{tabular}

Source: union communale des producteurs (UCP) Dassa-Zoumé, 2005.

\section{Ressources collectives des groupements villageois et des unions communales de producteurs}

En zones cotonnières, les ressources collectives des groupements villageois (GV) et des unions communales de producteurs (UCP) sont constituées essentiellement des frais de prestation dans les opérations de distribution des intrants agricoles (engrais, insecticide...) et dans celles de commercialisation primaire du coton-graine. Les ressources des GV sont aussi constituées dans certaines localités des ristournes cotonnières qui représentent les valeurs monétaires des écarts de poids constatés entre la pesée au village et la commercialisation sortie d'usine. Certains GV utilisent ces ristournes comme fonds d'investissement collectif. D'autres, au contraire, les répartissent entre leurs membres au prorata des quantités individuelles de coton produites par ceux-ci.

Les prestations des organisations paysannes sur les intrants sont facturées à 2,5 FCFA par kilogramme d'engrais et à 5 FCFA par litre d'insecticide. A DassaZoumé, les frais de prestation sur les intrants sont équitablement répartis entre le GV et l'union communale de producteur (voir le tableau B, en page suivante). 


\section{Tableau B}

Frais moyens de prestation intrants des GV et des UCP

\begin{tabular}{|l|r|r|r|r|r|r|}
\cline { 2 - 7 } \multicolumn{1}{c|}{} & \multicolumn{3}{|c|}{ Prestations sur engrais } & \multicolumn{3}{|r|}{ Prestations sur insecticide } \\
\cline { 2 - 7 } & $\begin{array}{r}\text { Quantité } \\
(\mathrm{kg})\end{array}$ & $\begin{array}{r}\text { Frais } \\
\text { de prestation } \\
\text { GV (FCFA) }\end{array}$ & $\begin{array}{r}\text { Frais } \\
\text { de prestation } \\
\text { UCP (FCFA) }\end{array}$ & $\begin{array}{r}\text { Quantité } \\
(\text { litre) }\end{array}$ & $\begin{array}{r}\text { Frais } \\
\text { de prestation } \\
\text { GV (FCFA) }\end{array}$ & $\begin{array}{r}\text { Frais } \\
\text { de prestation } \\
\text { UCP (FCFA) }\end{array}$ \\
\hline Petit GV & 6500 & 13000 & 13000 & 65 & 162 & 162 \\
\hline GV moyen & 60000 & 75000 & 75000 & 2104 & 5260 & 5260 \\
\hline Gros GV & 400000 & 500000 & 500000 & 5800 & 14500 & 14500 \\
\hline
\end{tabular}

Source: UCP Dassa-Zoumé, 2005.

Les frais de prestation payés aux GV dans le cadre des activités de commercialisation primaire s'élèvent à 1725 FCFA par tonne de coton-graine. Ceux payés à l'union communale des producteurs sont de l'ordre de 2875 FCFA par tonne. Sur ces frais de prestation payés à l'UCP, l'union départementale des producteurs perçoit 300 FCFA par tonne de coton-graine commercialisé pour le financement de son fonctionnement.

Sur la base de ces considérations, les recettes de différents types de GV sont estimées au tableau C.

Les recettes de prestation de l'UCP sur la commercialisation du coton-graine peuvent être estimées à titre indicatif dans la commune de Dassa-Zoumé au titre de la campagne agricole 1997-1998. La production cotonnière de ce territoire en cette année étant estimée à 25000 tonnes, les prestations de l'UCP dans la commercialisation primaire du coton-graine ont engendré une recette totale de 2875 x 25000 , soit 71875000 FCFA.

\section{Tableau C}

Recettes de prestations de différents types de GV dans le cadre de la commercialisation primaire du coton-graine

\begin{tabular}{|l|r|l|}
\hline Type de GV & $\begin{array}{l}\text { Production moyenne de coton-graine } \\
\text { (tonnes) }\end{array}$ & Frais de prestation (FCFA) \\
\hline Petit GV & 10 & 17250 \\
\hline GV moyen & 300 & 517500 \\
\hline Gros GV & 2000 & 3450000 \\
\hline
\end{tabular}

Source: calcul sur la base des données collectées auprès de l'UCP Dassa-Zoumé (2005). 